\title{
An elF4E-interacting peptide induces cell death in cancer cell lines
}

\author{
M Masse ${ }^{1,2,3,7}$, V Glippa ${ }^{1,2,3,7}$, H Saad ${ }^{1,2,3,4}$, R Le Bloas ${ }^{3,4}$, I Gauffeny ${ }^{1,2,3}$, C Berthou ${ }^{3,4}$, M Czjzek $^{3,5,6}$, P Cormier ${ }^{1,2,3}$ and B Cosson ${ }^{\star, 1,2,3,8}$
}

The eukaryotic initiation factor eIF4E is essential for cap-dependent initiation of translation in eukaryotes. Abnormal regulation of elF4E has been implicated in oncogenic transformation. We developed an elF4E-binding peptide derived from Angel1, a partner of elF4E that we recently identified. We show here that this peptide fused to a penetratin motif causes drastic and rapid cell death in several epithelial cancer cell lines. This necrotic cell death was characterized by a drop in ATP levels with F-actin network injury being a key step in extensive plasma membrane blebbing and membrane permeabilization. This synthetic elF4E-binding peptide provides a candidate pharmacophore for a promising new cancer therapy strategy.

Cell Death and Disease (2014) 5, e1500; doi:10.1038/cddis.2014.457; published online 30 October 2014

The eukaryotic initiation factor elF4E is essential for capdependent initiation of translation in eukaryotes and is considered to be the rate-limiting factor of protein synthesis. ${ }^{1}$ elF4E binds to the $5^{\prime}$ cap structure found on all nuclearencoded mRNAs and to the scaffolding protein elF4G, which along with elF3, bridges mRNA to ribosomes. ${ }^{2}$ elF4E and elF4G form the elF4F complex with elF4A, an ATP-dependent RNA helicase that facilitates ribosomal scanning from the $5^{\prime}$ cap by unwinding secondary structures within the $5^{\prime}$ untranslated region (5'UTR). elF4E also has a function in gene expression unrelated to translation initiation. It regulates the export of specific mRNAs, including cyclin D1, from the nucleus to the cytoplasm. 3,4

elF4E is regulated at multiple levels, including through interactions with a family of elF4E-binding proteins that compete with elF4G to bind to the dorsal face of elF4E. Hypophosphorylated elF4E binding protein 4E-BP1, the bestcharacterized inhibitor of elF4E activity, sequesters elF4E and prevents the recruitment of elF4G to the $5^{\prime}$ cap of mRNAs. Upon mitogen activation or stimulation with growth factors or cytokines, 4E-BP1 is phosphorylated at multiple sites by the mammalian target of rapamycin (mTOR) signaling pathway leading to its dissociation from elF4E. ${ }^{5}$ Accordingly, elF4E activity has been linked to growth stimulation and oncogenic transformation that enhance the translation of a subset of mRNAs believed to be poorly expressed in normal cellular conditions. These mRNAs predominantly encode growth factors and proto-oncogenes involved in cell proliferation and promote tumor cell survival, angiogenesis, transformation, invasion and metastasis. ${ }^{6}$

Cancer cells frequently show elevated levels of elF4E, ${ }^{7}$ reduced expression of $4 \mathrm{E}-\mathrm{BP} 1$ and activation of signaling pathways that phosphorylate $4 \mathrm{E}-\mathrm{BP} 1{ }^{8}$ Elevated levels of elF4E are sufficient to induce deregulated growth and malignant transformation of a variety of cultured cell lines. ${ }^{9}$ Correlatively, overexpression of 4E-BP has been reported to partially reverse transformation mediated by the oncogenic gene $\mathrm{V}$-src. ${ }^{10}$ Targeting elF4E-elF4G interactions is a potential way to reverse the aberrant activation of elF4E in cancer. ${ }^{11}$ The small molecule inhibitor $4 \mathrm{EGI}-1$ and an elF4Ebinding peptide were described previously to inhibit growth and to have proapoptotic activities. ${ }^{12,13}$ We previously identified Angel1 as a new partner of elF4E and we showed that Angel1 efficiently competes with elF4G to bind to elF4E. ${ }^{14}$ In the present paper, we generated a new elF4Einteracting peptide designed from the elF4E-binding motif of Angel 1 to target elF4E-elF4G interactions. We demonstrate that this peptide can efficiently inhibit in vitro translation. Surprisingly, it also induces rapid cell death in a wide variety of cancer cell lines involving a dramatic disorganization of the F-actin network, cell blebbing and plasma membrane rupture.

\footnotetext{
${ }^{1}$ Sorbonne Universités, UPMC Univ Paris 06, UMR 8227, Integrative Biology of Marine Models, Translation Cell Cycle and Development, Station Biologique de Roscoff, CS 90074, Roscoff cedex, France; ${ }^{2}$ CNRS, UMR 8227, Integrative Biology of Marine Models, Station Biologique de Roscoff, CS 90074, Roscoff cedex, France; ${ }^{3}$ Université Européenne de Bretagne, Brittany, France; ${ }^{4}$ Institut de Cancéro-hématologie, Département d'Hématologie, Hôpital Morvan, CHRU, Brest, France; ${ }^{5}$ Sorbonne Universités, UPMC Univ Paris 06, UMR 8227, Integrative Biology of Marine Models, Végétaux marins et biomolécules, Station Biologique de Roscoff, CS 90074, Roscoff cedex, France and ${ }^{6} \mathrm{CNRS}$, UMR 8227, Integrative Biology of Marine Models, Végétaux marins et biomolécules, Station Biologique de Roscoff, CS 90074, Roscoff cedex, France

*Corresponding author: B Cosson, Epigenetics and Cell Fate, UMR 7216, Univ Paris Diderot, 35 rue Helène Brion, F-75013 Paris, France. Tel: +33 157278966 ; Fax: +33 1572789 12; E-mail: bertrand.cosson@ univ-paris-diderot.fr

${ }^{7}$ These authors contributed equally to this work.

${ }^{8}$ Present address: Epigenetics and Cell Fate, UMR 7216, Univ Paris Diderot, Paris F-75013, France.

Abbreviations: elF, eukaryotic initiation factor; ATP, adenosine triphosphate; F-actin, filamentous actin; 4E-BP, 4E-binding protein; mTOR, mammalian target of rapamycin; A1, Angel1; YFP, yellow fluorescent protein; PI, propidium iodide; DMSO, dimethyl sulfoxide; DIC, differential interference contrast; PARP, poly ADP ribose polymerase; RIP, receptor interacting protein; ROS, reactive oxygen species; $\mathrm{m}^{7} \mathrm{GTP}, 7$-methylguanosine $5^{1}$-triphosphate; IGF-I, insulin-like growth factor-1; c-FLIP, cellular FLICE (FADD-like IL-1 $\beta$-converting enzyme)-inhibitory protein; TRAIL, tumor necrosis factor-related apoptosis-inducing ligand; DR, death receptor; MCL1, myeloid cell leukemia 1

Received 24.2.14; revised 09.9.14; accepted 16.9.14; Edited by Amarante-Mendes
} 


\section{Results}

Generation of elF4E-interacting peptides. We recently characterized a new elF4E-interacting partner, Angel1. ${ }^{14}$ The interaction site of Angel1 (designated A1) contains the consensus $\mathrm{Y}-\mathrm{X}-\mathrm{X}-\mathrm{X}-\mathrm{X}-\mathrm{L}-\phi$ recognition motif (where $\mathrm{X}$ is variable and $\phi$ is an hydrophobic residue, usually $L, M$ or $F$ ) conserved in the 4E-BP and elF4G families throughout evolution and described to be essential for their binding to elF4E ${ }^{15,16}$ (for Angel1, see Supplementary Figure S3 in Gosselin et $a .^{14}$ ). We thus used this sequence to generate a new elF4E-binding peptide. An 11 amino acid sequence derived from the recognition motif of Angel1 (from -3 to +8 , annotated from the conserved tyrosine of the consensus motif, Figure 1a) was fused to the yellow fluorescent protein (YFP) as a carrier. This fusion protein, named YFP-A1 (for YFP-Angel1 peptide), was clearly retained on an elF4E column, but not YFP alone (Supplementary Figure S1,

a

\begin{tabular}{|c|c|c|}
\hline & $\begin{array}{ll}-3 & 0 \\
\end{array}$ & +13 \\
\hline & \multicolumn{2}{|c|}{ 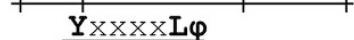 } \\
\hline A1 & \multicolumn{2}{|c|}{ RRKYGRDFLLRF } \\
\hline IRS & \multicolumn{2}{|c|}{ RYIRS } \\
\hline A1m & \multicolumn{2}{|c|}{ RRKAGRDFAARF } \\
\hline $\mathrm{A} 1-\mathrm{IRS}$ & \multicolumn{2}{|c|}{ RRKYGRDFLLRFRYIRS } \\
\hline Alm-IRS & \multicolumn{2}{|c|}{ RRKAGRDFAARFRYIRS } \\
\hline $\mathrm{A} 1-5 \mathrm{~A}$ & \multicolumn{2}{|c|}{ RRKYGRDFLLRFAAAAA } \\
\hline BP2-IRS & \multicolumn{2}{|c|}{ RIIYDRKFLLDRRYIRS } \\
\hline BP $2 m-I R S$ & \multicolumn{2}{|c|}{ RIIADRKFAADRRYIRS } \\
\hline
\end{tabular}

b

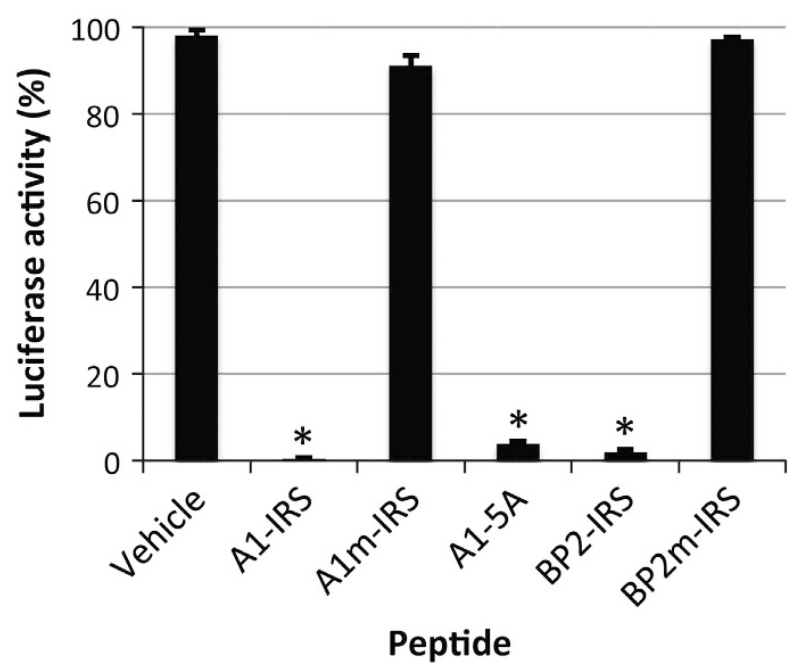

Figure 1 A1-IRS peptide inhibits in vitro translation. (a) Sequences of the elF4Ebinding motif of Angel1 (A1), the elF4E-binding protein 4E-BP2 (BP2), the penetratin IRS domain (IRS) and the synthesized peptides (A1-IRS, A1m-IRS, A1-5 A, BP2-IRS, BP2m-IRS). The consensus elF4E-binding motif $Y x x x x L \varphi$ is indicated. (b) Capped and polyadenylated Renilla luciferase mRNA was translated in rabbit reticulocyte lysate in the presence of $50 \mu \mathrm{M}$ of the indicated peptides. Renilla luciferase activity was then determined as described in Materials and Methods. Translation with the vehicle (DMSO) was arbitrarily set to $100 \%$. The results presented are representative of three independent experiments performed in triplicates and expressed as mean \pm S.D. Versus group control (vehicle): * $P<0.001$ compare lanes 5 and 2). Mutations affecting the hydrophobic amino acids tyrosine and leucine greatly decreased binding to elF4E (Supplementary Figure S1, lane 8), an expected result because these mutations in the elF4E-binding site of 4E-BP1 preclude binding to elF4E in chordates. ${ }^{16,17}$

$\mathrm{A} 1$ and the elF4E-binding domain of 4E-BP2 (designated BP2 herein) were fused to the IRS-penetratin peptide domain ${ }^{18}$ (giving rise to A1-IRS and BP2-IRS, respectively, Figure 1a) to allow internalization in cultured cells (see below). An elF4E-interacting peptide derived from 4E-BP has been shown to inhibit translation initiation; ${ }^{19}$ we therefore tested whether A1-IRS is also able to inhibit protein synthesis in an in vitro translation system. A1-IRS was as efficient as BP2-IRS in drastically inhibiting translation (Figure 1b), whereas, as expected, the consensus motif mutants did not affect translation activity (A1m-IRS and BP2m-IRS, Figure 1b). The A1-5A variant, obtained by replacing the IRS sequence with alanines, still inhibited translation activity (A1-5A, Figure $1 \mathrm{~b}$ ), indicating that the IRS-penetratin sequence had no role in translation inhibition. We validated by ${ }^{35} \mathrm{~S}$ Methionine incorporation that A1-IRS inhibits translation in living cells (Supplementary Figure. S2). We concluded that A1-IRS is a bona fide elF4E-inhibiting peptide.

A1-IRS induces cell blebbing that leads to cell death. We fused the A1 peptide to the IRS sequence to allow cellular uptake. The synthetic fusion peptide A1-IRS was efficiently taken up by HeLa cells (Supplementary Figure S3). HeLa cells loaded with the membrane-permeant green fluorescent Syto 13 dye were exposed to A1-IRS in the presence of the membrane-impermeant dye propidium iodide (PI). ${ }^{20}$ Cell fluorescence was monitored by microscope (Figure 2a), and cell viability was quantified (Figure $2 b$ ). Surprisingly, after a 30 min incubation with A1-IRS, most cells had incorporated the red fluorescent dye (Figures $2 a$ and $b$ ). The PI staining was characteristic of membrane permeability and cell death. As expected, we observed loss of membrane integrity upon prolonged incubation with A1-IRS (data not shown). Importantly, cells incubated with the peptide variant unable to bind to elF4E (A1m-IRS) were not permeable to $\mathrm{PI}$ and retained the green fluorescence (Figures $2 a$ and $b$ ), as observed for the control, dimethyl sulfoxide (DMSO)-treated cells (vehicle, Figure 2b). As expected, the variant without the IRS-penetratin sequence had no effect on cell viability (A1$5 A$, Figure $2 b$ ). These results indicate that the peptide must contain a functional elF4E-binding motif to induce cell death. However, incubation with BP2-IRS did not induce cell death (Figure 2b). Therefore, the specific sequence of the Angel1 elF4E-binding motif is necessary to induce cell death.

Under differential interference contrast (DIC) microscopy, cells showed peculiar morphology after incubation with A1IRS (Figure 2a). DIC illumination gives a pseudo threedimensional appearance to the specimen that does not reflect the real cell shape, but nonetheless indicates regions of different thicknesses and/or refractive index. After treatment with A1-IRS, the nucleus was delineated by a ring-like structure that we assumed to be the nuclear envelope, given the PI staining (Figure 2a). The nucleus contained spherical granules that probably correspond to nucleoli. To further explore these morphological changes, we performed 

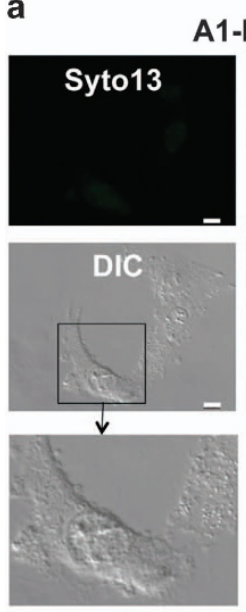

A1-IRS
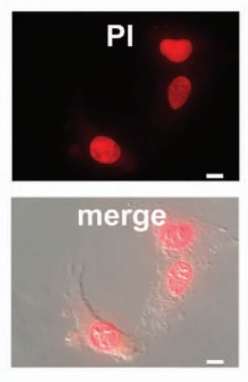

b

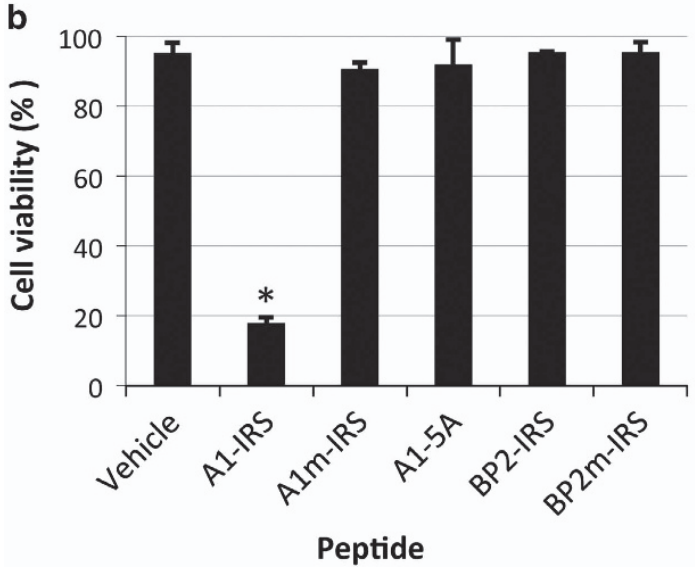

A1m-IRS
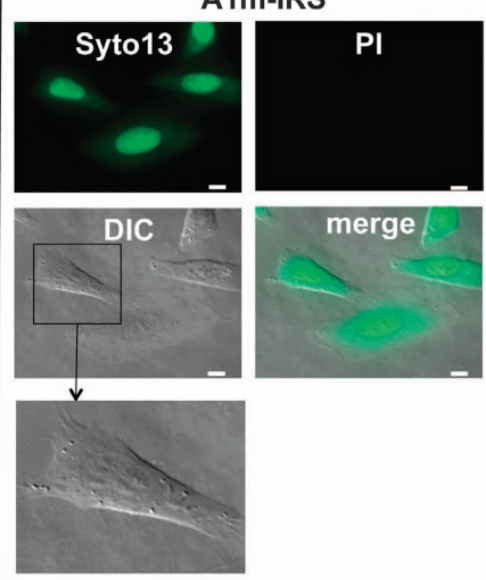

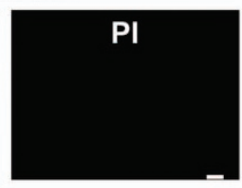

merge

C

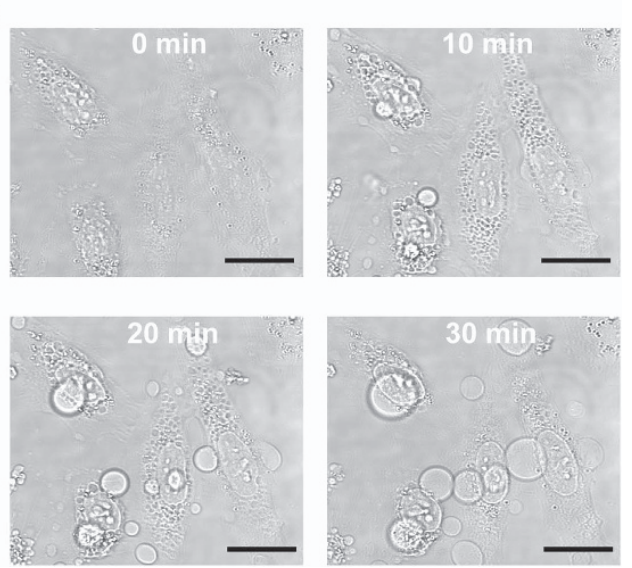

Figure 2 A1-IRS induces membrane blebbing and HeLa cell death as evaluated by plasma membrane permeability. HeLa cells were incubated with $50 \mu \mathrm{M}$ of the indicated synthesized peptides or with the vehicle (DMSO) control. (a) HeLa cells were incubated 30 min with A1-IRS (left panel) or A1m-IRS (right panel), in presence of $5 \mathrm{mM}$ Syto 13 and $12.5 \mu \mathrm{g} / \mathrm{ml}$ propidium iodide (PI). Images were collected on a Zeiss observer Z1 Fluorescence microscope using a $\times 63$ objective. Scale bar $=25 \mu \mathrm{m}$. (b) Cell viability was determined as the percentage of PI-negative and Syto 13-positive cells using the ImageJ program. At least 200 cells per well were counted. The data are given as the mean \pm S.D. of triplicates, and are representative of three experiments. Versus group control (vehicle): ${ }^{*} ; P<0.001$. (c) A field of HeLa cells were imaged under phase contrast every 10 min using a Leica SP5 microscope with a $\times 63$ objective. The recording begins 2 min after adding A1-IRS in the cell culture medium. Scale bar $=25 \mu \mathrm{m}$

a time-lapse experiment. As shown in Figure 2c, incubation with A1-IRS induced massive cell blebbing, with the diameter of the blebs reaching a diameter similar to that of the nucleus. Assuming that the total cell volume is conserved, the volume and the thickness of the cell decrease with bleb growth. Consequently, cell blebbing may induce differences in the refractive index of the cell structures, explaining the specific DIC pattern observed after incubation with A1-IRS (Figure 2a).

To examine the relationship between bleb growth and cell permeabilization, both were simultaneously recorded in a HeLa cell. Blebs first appeared and reached a substantial size before cell permeabilization was observed with PI staining of the nucleus (Supplementary Movie 1). Blebs did not continue to increase after membrane permeabilization. In summary, we observed that the A1-IRS peptide caused massive cell blebbing that precedes membrane permeabilization and cell death.

A1-IRS causes cell death in various cell lines. The A1-IRS peptide showed cytotoxic activity in HeLa cells that are derived from a cervix adenocarcinoma. We therefore evaluated the cytotoxicity of A1-IRS in other cancer cell lines established from mammary gland ductal carcinoma (MDA), gastric adenocarcinoma (HGT1), cervix adenocarcinoma (HeLa), melanoma (Skmel), colorectal carcinoma (HCT116), chronic lymphocytic leukemia (JOK-1) and from adenovirus transformed epithelial embryonic kidney cells (HEK-293). Cells were incubated for $60 \mathrm{~min}$ with 10-50 $\mu \mathrm{M}$ A1-IRS. Cell death was then assessed by double staining with Syto 13 and PI. Membrane permeabilization was efficiently induced by A1-IRS in all tested cell lines (Figure 3), but, as expected, not by the A1m-IRS variant peptide (data not shown). However, cell lines differed in sensitivity: chronic B-lymphocytic leukemia JOK-1 cell lines that grow in suspension showed the highest sensitivity, whereas the embryonic kidney cell line was only moderately affected by the peptide, with less than $40 \%$ of cell death observed at $50 \mu \mathrm{M}$ A1-IRS (Figure 3). Given that JOK-1 cells are grown in suspension culture and the other cell types are adherent culture, we tested whether culture type changes the sensitivity of the response to 


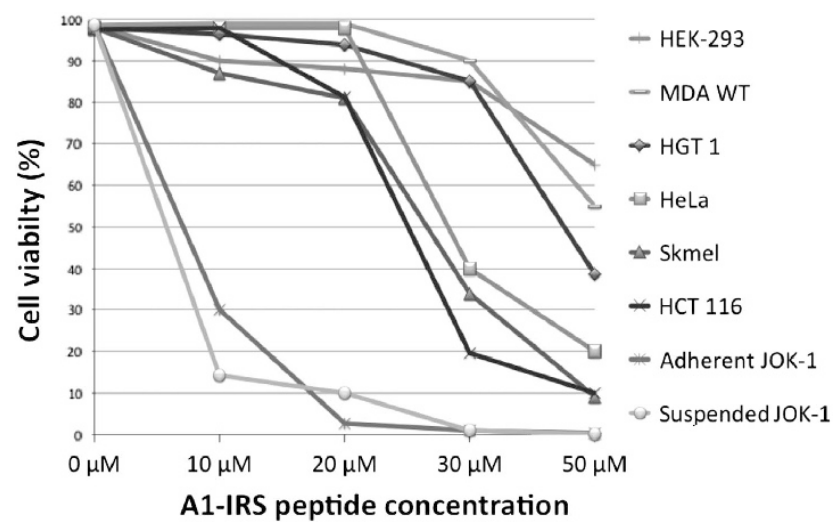

Figure 3 A1-IRS induces cell death in various cell lines. Cell cultures were incubated with increasing concentrations of the synthetic A1-IRS peptide for $60 \mathrm{~min}$ and cell death was determined as described in Figure $2 b$. Results are expressed as the mean of three independent experiments. HEK-293, adenovirus-transformed epithelial embryonic kidney cells; MDA, mammary gland ductal carcinoma; HGT1, gastric adenocarcinoma; HeLa, cervix adenocarcinoma; Skmel, melanoma; HCT116, colorectal carcinoma; JOK-1, chronic lymphocytic leukemia

the peptide. In suspension culture, cells are uniformly exposed to the culture medium, whereas in adherent cultures, cells are in a monolayer and are only exposed on one side. JOK-1 cells incubated in a serum-free medium for $12 \mathrm{~h}$ became adherent, $10 \%$ serum was then added and they were subsequently cultured $24 \mathrm{~h}$ before assessing the cytotoxic activity of the peptide. As shown in Figure 3, adherent JOK-1 cells showed an A1-IRS dose-response profile similar to that of JOK-1 cells in suspension, indicating that the pattern of JOK-1 sensitivity was not due to culture conditions, but due to the intrinsic characteristics of the cell line. Given the maximum sensitivity of JOK-1 cells to A1-IRS, we conducted all subsequent experiments on this cell line.

\section{A1-IRS cytotoxic activity does not require active transla-} tion, but depends on elF4E availability. In HeLa cells, only A1-IRS induced cell death, and not BP2-IRS (Figure 2b). We observed a similar pattern of cell viability for JOK-1 cells (Figure 4a), indicating that not only is the specific sequence of the Angel1 elF4E-binding motif necessary to induce cell death but also it does not depend on the cell line. Although A1-IRS and BP2-IRS were both able to inhibit in vitro translation, they showed differences in cytotoxicity, which may possibly be due to translation repression by A1-IRS of specific messenger mRNA. If so, a general inhibitor of translation should cause cell death to occur. Preincubation of JOK-1 cells with emetine, a translation inhibitor, for $1 \mathrm{~h}$ induced translation arrest, as attested by the absence of ${ }^{35} \mathrm{~S}$ met incorporation. This translation arrest was not sufficient to induce cell death after $1 \mathrm{~h}$ (Supplementary Figure S4) and even after $4 \mathrm{~h}$ of incubation (data not shown). Emetine-pretreated cells were incubated with A1-IRS, and cell death was efficiently induced (Supplementary Figure S4). We conclude that A1-IRS induces cell death independently of translation activity.

As the cytotoxic activity of A1-IRS depends on its elF4Ebinding motif, we looked for the relationships between A1-IRS and elF4E. Various partners of elF4E-including Angel1, elF4G and 4E-BP proteins-use the same binding motif and compete with each other to bind to elF4E. ${ }^{14}$ When we purified elF4E and its associated partners from cells incubated with A1-IRS, A1-IRS displaced the elF4G-elF4E interaction (Figure 4b, lane 5). As expected, this effect was not observed with the mutated peptide A1m-IRS that cannot bind to elF4E (Figure 4b, lane 6). Therefore, A1-IRS can interfere with elF4E complexes in cells.

We then tested whether elF4E availability was a necessary prerequisite for the cytotoxic effect of A1-IRS. Cells were preincubated with a high amount of BP2-IRS $(200 \mu \mathrm{M})$ to sequester elF4E before adding A1-IRS. Although a $1 \mathrm{~h}$ preincubation with BP2-IRS did not cause membrane permeabilization in JOK-1 cells in the absence of peptide addition (Figure 4a), preincubation with BP2-IRS decreased the cytotoxic effect of A1-IRS (Figure 4c). Co-incubation with the control peptide BP2m-IRS, which cannot bind to elF4E, did not significantly affect A1-IRS activity. The cytotoxic effect of A1IRS therefore requires elF4E availability.

A1-IRS causes necrosis through actin disorganization. We performed a set of experiments, summarized in Table 1, to characterize the mechanism of cell death mediated by A1IRS. Intracellular ATP levels have been implicated both in vitro and in vivo as a determinant of the mechanism of cell death. ${ }^{21}$ We observed a rapid decrease in ATP levels in JOK- 1 cells 10 min after addition of A1-IRS (Supplementary Figure S5). We observed by simultaneous monitoring of the release of adenylate kinase as a marker of cell membrane permeabilization (see Materials and Methods), that drop in ATP and cytolysis were concomitant (Supplementary Figure S5). Membrane blebs are typical of necrosis, they grew without retraction and reached diameters of up to several tens of micrometers, followed by plasma membrane permeabilization to $\mathrm{PI}$ and release of cytosolic proteins. ${ }^{22}$ As there are no absolute criteria to unequivocally identify necrosis, this type of cell death must be confirmed by eliminating the possibility of the occurrence of apoptosis, autophagy or other 'atypical' cell death modalities, as established by the Nomenclature Committee on Cell Death. ${ }^{23}$ The caspase inhibitor ZVAD-FMK did not inhibit AI-IRS cytotoxicity, and there was no PARP, caspase 3 or caspase 8 cleavage after incubation with A1-IRS (Supplementary Figures S6 and S7), indicating that these proteins were not activated. We did not observe any nuclear fragmentation under optical (Figure 2) or electronic microscopy (data not shown). Inhibition of phospholipase C did not affect the cytotoxicity of A1-IRS (Supplementary Figure S8). We also excluded cell death due to autophagy because the inhibitor 3-methyladenine did not inhibit A1-IRS cytotoxic activity (Supplementary Figure S6) and because there were no double membrane autophagic vacuoles under electronic microscopy (data not shown). Inhibition of RIP1 by necrostatin-1 did not inhibit A1-IRS activity, thereby excluding necroptosis (Supplementary Figure S9). ${ }^{24}$ We also looked for mitochondrial perturbation and did not observe any cytochrome C redistribution (Supplementary Figure S10) or ROS increase (Supplementary Figure S11) after incubation with A1-IRS, and mitochondria appeared normal when 
a

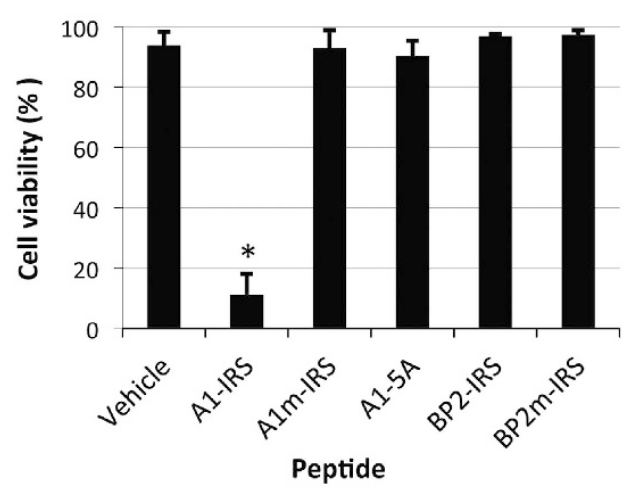

C

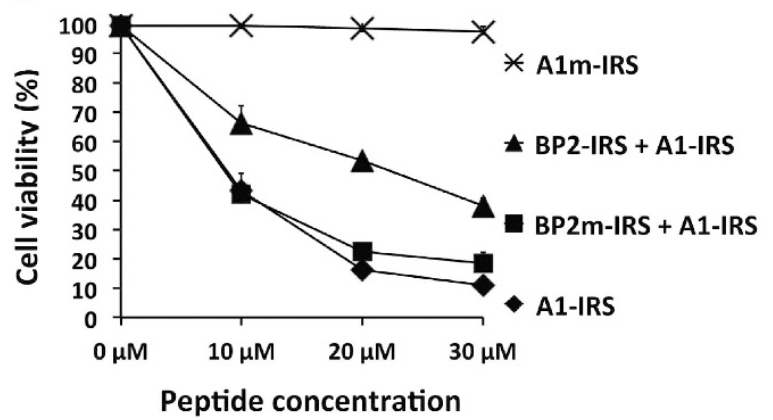

b

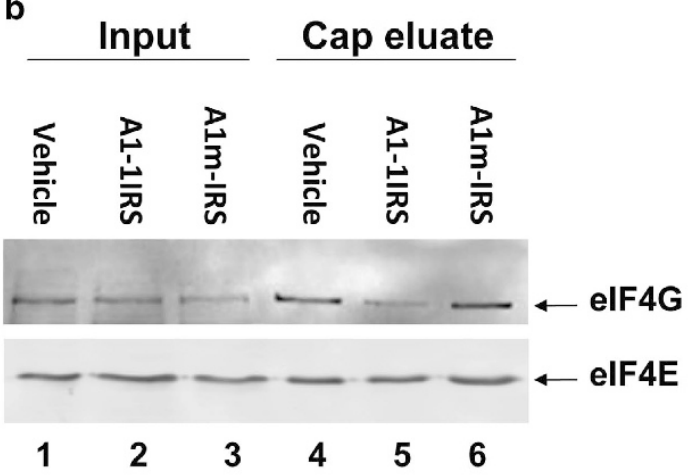

d

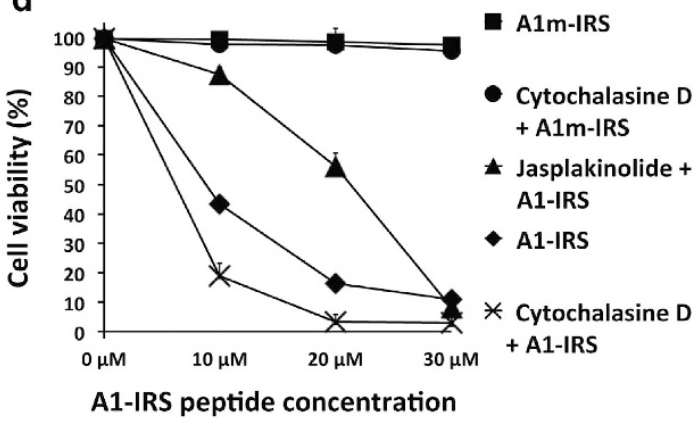

Figure 4 A1-IRS cytotoxicity requires elF4E availability and causes necrosis via F-actin destabilization. (a) JOK-1 cells were incubated with $50 \mu \mathrm{m}$ of the indicated peptides and analyzed as described for HeLa cells in Figure 2b. The data are given as the mean \pm S.D. of triplicates, and are representative of three experiments. Versus group control (vehicle): *; $P<0.001$. (b) JOK-1 cells were incubated for $1 \mathrm{~h}$ with A1-IRS, A1m-IRS or the vehicle (DMSO). Protein extracts were incubated with $\mathrm{m}^{7} \mathrm{GTP}$ beads to purify elF4E and its associated factors; eluted proteins were analyzed by western blot with antibodies against elF4E and elF4G. (c) Increasing concentrations (from 0 to $30 \mu \mathrm{M}$ ) of A1-IRS or A1m-IRS peptides were added in JOK-1 cells that were previously incubated with $200 \mu \mathrm{M}$ BP2-IRS or BP2m-IRS for $1 \mathrm{~h}$. After 10 min, cell viability was determined as described in a. Results are representative of three independent experiments. (d) JOK-1 cells were pre-treated with $0.5 \mathrm{ng} / \mu \mathrm{l}$ cytochalasin D for $1 \mathrm{~h}$, or with jasplakinolide for $2 \mathrm{~h}$. A1-IRS or A1m-IRS was then added at the indicated concentration and cell viability was determined after 10 min as described in (a). Results are representative of three independent experiments

observed under electronic microscopy (data not shown). The cytosolic concentration of calcium did not increase (Supplementary Figure S12) and cathepsin inhibition (Supplementary Figure S13) did not impede A1-IRS activity. Taken together, these results indicate that we did not observe the properties of any specific cell death modality. We therefore concluded that the observed cell death is a necrotic cell death characterized by massive cell blebbing, leading to an early loss of integrity of the plasma membrane and a drop in ATP levels.

Blebs generally appear when the actin links between the plasma membrane and the underlying cytoskeleton are weakened. ${ }^{25}$ Because the actin cytoskeleton network is also implicated in cell death regulation in a wide variety of cells, ${ }^{26}$ we tested whether actin dynamics have a role in the cytotoxicity of A1-IRS. We preincubated cells with cytochalasin D or jasplakinolide, which respectively, prevent actin polymerization and inhibit depolymerization of actin microfilaments, prior to the addition of A1-IRS. As expected, cytochalasin $D$ treatment alone had no effect on PI permeabilization (Figure 4d), but pre-incubation with cytochalasin D potentialized A1-IRS cytotoxicity as shown by a doseresponse experiment (Figure 4d). Conversely, jasplakinolide had the opposite effect, resulting in a decreased efficiency of
A1-IRS to mediate cell death (Figure 4d). To investigate the cellular distribution of actin, we performed immunofluorescence experiments on HeLa cells because our attempts to use this technique for JOK-1 cells were unsuccessful. We observed a dramatic disorganization of F-actin microfilaments in cells incubated with A1-IRS (Supplementary Figure S14). Examination of protein levels using western blotting showed that $\mathrm{B}$-actin was unaffected by A1-IRS treatment in both JOK-1 (Supplementary Fig. S7) and HeLa cells (not shown). Taken together, these experiments demonstrate that actin network destabilization is a necessary step for the cytotoxic effect of A1-IRS.

\section{Discussion}

Here, we generated an original peptide, A1-IRS, derived from Angel1, a partner of elF4E that we recently identified. ${ }^{14}$ The A1-IRS peptide interacts with elF4E, inhibits in vitro translation and, surprisingly, provokes rapid and massive cell death in a wide variety of cancer cell lines. The induced necrosis is characterized by a drop in ATP levels, with F-actin network injury being a key step to the rupture of the plasma membrane. This cytotoxicity was not due to the general physicochemical properties of A1-IRS, as this non-amphipathic peptide 
Table 1 List of the experiments conducted and the drugs used to characterize the cytotoxicity of A1-IRS

\begin{tabular}{llcc}
\hline Analysis & Experiment & Figure & Result \\
\hline Microscopy & PI staining detection & 2 & + \\
Luminescence & Drop in ATP levels detection & S5 & + \\
Microscopy & Nuclear fragmentation & 2 & - \\
Western blot & Cetection & & - \\
Western blot & Caspase 3 cleavage detection & S7 & - \\
Western blot & PARP cleavage detection & S7 & - \\
Western blot & MCL1 cleavage detection & S7 & - \\
Western blot & Cytochrome C release & S10 & - \\
Microscopy & ROS increase & S11 & - \\
Microscopy & Calcium increase with & S12 & - \\
& Fluo4-AM probe & & \\
\hline Reagent & Target & Figure & Cytotoxicity \\
& & & of A1-IRS \\
\hline ZVAD-fmk & Caspases & S6 & - \\
U73122 & Phospholipase C & S8 & - \\
3-methyladenine & Autophagic cell death & S6 & - \\
Necrostatin-1 & Rip1 & S9 & - \\
CA-074Me & Cathepsin & S13 & - \\
Emetine & Translation & S4 & - \\
4E-BP2 peptide & elF4E & $4 \mathrm{C}$ & Slowed \\
Cytochalasin D & Actin F destabilization & $4 d$ & Accelerated \\
Jasplakinolide & Actin F stabilization & $4 \mathrm{~d}$ & Slowed \\
& & & \\
\hline & & &
\end{tabular}

displays similar charge and hydrophobicity profiles as its inactive counterpart A1m-IRS (data not shown).

Through its binding to elF4E, A1-IRS competes elF4G (Figure $4 \mathrm{~b}$ ) and is able to inhibit translation (Figure $1 \mathrm{~b}$ and Supplementary Figure S2). However, in the absence of translation, rapid plasma membrane permeabilization still occurred upon incubation with A1-IRS, indicating that translation initiation activity of elF4E is not the cause of cell death induced by the peptide. Although we cannot formally exclude the possibility that the partial inhibition of translation induced by A1-IRS in living cells (Supplementary Figure S2) is an essential prerequisite for cell death, the rapid change in cell permeability seems to happen too quickly to be due to inhibition of protein synthesis.

As BP2-IRS did not cause cell death, the elF4E-binding property of a peptide is not sufficient to induce cell death. Therefore, it remains possible that A1-IRS is bound to molecular targets other than elF4E to mediate cell death. However, BP2-IRS preincubation decreased the cytotoxic activity of A1-IRS (Figure 4c), suggesting that elF4E must be available for A1-IRS to produce its cytotoxic effect. Furthermore, in addition to A1m-IRS, two other point mutations expected to decrease binding to elF4E showed a reduced capacity to inhibit in vitro translation and mediate cell death (data not shown). Thus, elF4E binding capacity and cytotoxic activity are tightly linked.

Earlier studies have established a possible link between elF4E and actin dynamics. elF4E associates with two different actin networks in dendrites; treatment with brain-derived neurotrophic factor increases the proportion of elF4E to levels similar to that located in dendritic spine heads consisting of a highly branched network of filaments. ${ }^{27}$ Furthermore, actin and elF4E have been co-purified by $\mathrm{m}^{7}$ GTP-Sepharose chromatography from migrating cell extracts. ${ }^{28}$ elF4E has also been found associated with actin-rich pseudopodia in six metastatic tumor cell lines, elF4E knockdown induced reduced F-actin density and slower loss of F-actin during treatment by the depolymerizing drug latrunculin $A .{ }^{29}$ Importantly, expression of a constitutively hypophosphorylated 4EBP1 sequesters elF4E and inhibits IGF-I-stimulated F-actin reorganization and lamellipodia formation. ${ }^{30}$ All of these findings are consistent with our result whereby F-actin was reorganized during incubation with A1-IRS, suggesting a functional link between elF4E and F-actin dynamics.

An elF4E-interacting peptide derived from BP2 fused to a penetratin sequence was previously shown to induce cell death, which shows characteristics of apoptosis and observed only with $72 \mathrm{~h}$ serum-starved cells. ${ }^{12}$ The small molecule 4EGI-1 that also inhibits elF4E-elF4G interaction mediates a TRAIL-mediated apoptosis through c-FLIP downregulation and DR5 induction. ${ }^{13,31}$ BP2 peptide-penetratin, 4EGI-1 and A1-IRS primarily target elF4E and induce cell deaths independently of translation inhibition. These results emphasize the need to further investigate the role of elF4E in the control mechanisms of cell survival.

Tumor-specific peptide agents hold great promise for clinical applications in cancer diagnosis and therapy. Our results show that A1-IRS may be a candidate pharmacophore to induce rapid cell death. The cell lines that we tested differed in sensitivity (Figure 3). Therefore, this cytotoxic peptide may have clinical utility and should be improved by selective targeting. The high sensitivity of JOK-1, a cell line established from a patient with chronic lymphocytic leukemia, suggests that A1-IRS may be an interesting tool for therapeutic strategies in this type of cancer. As antibody fusion could be used as a specific tool for cell targeting, work is currently underway to evaluate whether an A1-IRS-antibody fusion is a useful approach.

\section{Materials and Methods}

Peptides and other reagents. Peptides were produced and purified by HPLC (purity $>98 \%$ ), before lyophilization (GeneCust, Dudelange, Luxembourg). Peptides were dissolved at $50 \mathrm{mM}$ in 99.8\% DMSO (Sigma, Saint-Quentin Fallavier, France), and then diluted to different concentrations in water or in culture medium for cell-based experiments. The final concentration of DMSO in cultures was always less than $0.4 \%(\mathrm{v} / \mathrm{v})$. Sequences of the peptides used are listed in Figure 1a. A translation grade ${ }^{35} \mathrm{~S}$-methionine was used (EasyTag Methionine L- $\left[{ }^{35} \mathrm{~S}\right]$, PerkinElmer, Waltham, MA, USA). ${ }^{32}$

Analysis of in vitro interactions between YFP-A1 and GSTeIF4E. After production and purification, GST-elF4E recombinant protein ${ }^{17}$ was dialyzed overnight in buffer A (50 mM HEPES pH 7.7, $150 \mathrm{mM} \mathrm{KCl,} 1 \mathrm{mM}$ EDTA, $5 \%$ glycerol). Then, $1 \mu \mathrm{g}$ of GST-elF4E was incubated for $1 \mathrm{~h}$ with $25 \mu \mathrm{l}$ of GSHSepharose beads in buffer A. After washing, the beads were incubated for $1 \mathrm{~h}$ in buffer A containing $1 \mathrm{mg} / \mathrm{ml}$ of bovine serum albumin, $0.5 \%$ Igepal with $10 \mu \mathrm{l}$ rabbit reticulocyte lysate (TnT T7 Coupled Reticulocyte Lysate System, Promega, Charbonnières, France) that was programmed with YFP, YFP-A1 or YFP-A1m PCR DNA (all without the sequence coding for the penetratin IRS domain, see text) in presence of $\left[{ }^{35} \mathrm{~S}\right]$ methionine. After extensive washing, the beads were boiled in Laemmli buffer and analyzed by autoradiography on a Typhoon Trio imager (Amersham Pharmacia Biotech, Uppsala, Sweden).

Translation assay in rabbit reticulocyte lysate. Capped mRNAs coding for Renilla luciferase (Luc R) were produced by a T7 Message Machine Kit system (Ambion, Life Technologies, Saint Aubin, France) from pGb-Eg2-410 $\Delta 2$ hxG-A65 EcoRV-linearized vector. ${ }^{33}$ RNA was then purified by phenol-chloroform extraction and controlled by gel electrophoresis. In vitro translation was carried out in the Flexi-rabbit reticulocyte system (RRL, Promega), supplemented with $20 \mathrm{mM}$ amino acids (Promega), $1 \mathrm{U} / \mu \mathrm{l}$ RNase inhibitor (Ambion), potassium chloride (KCl), and $5 \mathrm{fmol}$ mRNA per reaction. Renilla activity from translation reaction was 
measured using the Renilla Luciferase Assay System (Promega) on a Tristar LB941 luminometer (Berthold, Thoiry, France).

Cell culture. JOK-1 cells were grown in complete RPMI 1640 medium with glutaMAX (Gibco BRL, Life Technologies) supplemented with 10\% heat-inactivated fetal calf serum, $1 \mathrm{unit} / \mathrm{ml}$ penicillin, $1 \mu \mathrm{g} / \mathrm{ml}$ streptomycin and $1 \% \mathrm{~L}$-glutamine, (Gibco BRL). For adherent cell cultures, cells were grown in complete Dulbecco's modified Eagle medium (Gibco BRL) with 10\% heat-inactivated fetal calf serum, 1 unit/ml penicillin, $1 \mu \mathrm{g} / \mathrm{ml}$ streptomycin and $1 \%$ L-glutamine (Gibco BRL). All cell lines were grown at $37^{\circ} \mathrm{C}$ in a $5 \% \mathrm{CO}_{2}$ humidified atmosphere.

Time-lapse microscopy. Cells were loaded in black 96 well plates with glass bottoms and 40000 cells per well. Before peptide treatment, $5 \mu \mathrm{M}$ Syto 13 (Molecular Probes, Gibco BRL) and $1 \mathrm{mg} / \mathrm{ml}$ propidium iodide (PI) (Sigma) were added to the culture medium. ${ }^{34}$ FM-464 (Molecular Probes, Gibco BRL) was used at $1 \mu \mathrm{M}$ before recording Supplementary Movie 1. Fluorescence was followed at various time intervals with a Zeiss Observer Z1 (Zeiss, Marly Le Roi, France) or a Leica TCS SP5 microscope (Leica, Nanterre, France). When indicated, cell morphology was followed by DIC and phase contrast microscopy.

Immunofluorescence microscopy. HeLa cells $\left(2 \times 10^{4}\right)$ were plated on 8 well Lab-Tek II Chamber Slides and grown overnight. After incubation with peptides or the vehicle (DMSO), cultures were washed three times with PBS, treated with BD Cytofix/Cytoperm kit (4\% paraformaldehyde with $0.1 \%$ saponin and $3 \%$ fetal calf serum (Cat No.554714 BD Biosciences, Le Pont de Claix, France) at $4{ }^{\circ} \mathrm{C}$ for $20 \mathrm{~min}$. Fixed and permeabilized cells were incubated with primary antibody diluted in BD Cytoperm/Wash solution. After overnight incubation at $4^{\circ} \mathrm{C}$, cells were washed and incubated with the appropriate secondary antibody at room temperature for $1 \mathrm{~h}$. Cells were washed three times (10 min each) in BD Perm/ Wash Buffer and stained with $1 \mu \mathrm{g} / \mathrm{ml}$ Hoechst 33342 (Sigma) in BD Perm/Wash Buffer for $10 \mathrm{~min}$. After two further washes, the slides were covered with a cover slip, using Fluoprep (BioMérieux, Lyon, France) as a mounting medium and sealed with clear nail polish. Images were collected on a Zeiss fluorescence microscope using $\mathrm{a} \times 63$ oil objective. Polyclonal rabbit antibodies raised against the $\mathrm{A} 1$ peptide RRKYGRDFLLC (P16; $1: 500)$ were used to localize the A1-IRS peptide. No signal was detected after incubating cells with A1m-IRS, presumably because of the failure of the antibody to recognize the mutated sequence. Secondary antibodies used were Cy 3-conjugated AffiniPure Donkey anti-mouse IgG (Jackson ImmunoResearch laboratories, West Grove, PA, USA) at a concentration of $1: 500$, or antirabbit conjugated to Alexa Fluor 488 (Molecular Probes) at a 1:500 dilution. F-Actin was visualized using a phalloidin-rhodamine conjugate (Molecular Probes), at a final concentration of $0.3 \mu \mathrm{M}$. Before observation under microscope, HeLa cells were washed in BD Perm/Wash Buffer with $1 \%$ bovine serum albumin.

Detection of ToxiLight AK and determination of ATP levels. JOK-1 cells were diluted to 30000 cells per well in $100 \mu$ l of culture medium, and were plated on 96-well plates. Each peptide was diluted in cell medium to reach the indicated concentration. After incubation with the peptide, analysis of adenylate kinase release was measured using the ToxiLight BioAssay Kit (Lonza, Levallois Perret, France), and ATP levels were determined with the CellTiter-Glo Luminescent Cell Viability Assay (Promega) according to the manufacturer's instructions in a Tristar LB941 luminometer (Berthold).

\section{Conflict of Interest}

The authors declare no conflict of interest.

Acknowledgements. This work was supported by the Ligue contre le Cancer, the Brittany Regional Council, and the fondation ARC pour la Recherche sur le Cancer. The HeLa cell line was used in this research. Henrietta Lacks, and the HeLa cell line that was established from her tumor cells without her knowledge or consent in 1951, have made significant contributions to scientific progress and advances in human health. We are grateful to Henrietta Lacks, now deceased, and to her surviving family members for their contributions to biomedical research.

1. Duncan R, Milburn SC, Hershey JW. Regulated phosphorylation and low abundance of HeLa cell initiation factor elF-4F suggest a role in translational control. Heat shock effects on elF-4F. J Biol Chem 1987; 262: 380-388.
2. Shatkin AJ. mRNA cap binding proteins: essential factors for initiating translation. Cell 1985; 40: 223-224.

3. Lai HK, Borden KL. The promyelocytic leukemia (PML) protein suppresses cyclin D1 protein production by altering the nuclear cytoplasmic distribution of cyclin D1 mRNA. Oncogene 2000; 19: 1623-1634.

4. Rousseau D, Kaspar R, Rosenwald I, Gehrke L, Sonenberg N. Translation initiation of ornithine decarboxylase and nucleocytoplasmic transport of cyclin D1 mRNA are increased in cells overexpressing eukaryotic initiation factor 4E. Proc Natl Acad Sci USA 1996; 93: 1065-1070.

5. Gingras AC, Raught B, Gygi SP, Niedzwiecka A, Miron M, Burley SK et al. Hierarchical phosphorylation of the translation inhibitor 4E-BP1. Genes Dev 2001; 15: 2852-2864.

6. Silvera D, Formenti SC, Schneider RJ. Translational control in cancer. Nat Rev Cancer 2010; 10: 254-266.

7. Graff JR, Konicek BW, Lynch RL, Dumstorf CA, Dowless MS, McNulty AM et al. elF4E activation is commonly elevated in advanced human prostate cancers and significantly related to reduced patient survival. Cancer Res 2009; 69: 3866-3873.

8. Huang S, Houghton PJ. Targeting mTOR signaling for cancer therapy. Curr Opin Pharmacol 2003; 3: 371-377.

9. Lazaris-Karatzas A, Montine KS, Sonenberg N. Malignant transformation by a eukaryotic initiation factor subunit that binds to mRNA $5^{\prime}$ cap. Nature 1990; 345: 544-547.

10. Rousseau D, Gingras AC, Pause A, Sonenberg N. The elF4E-binding proteins 1 and 2 are negative regulators of cell growth. Oncogene 1996; 13: 2415-2420.

11. Cencic R, Hall DR, Robert F, Du Y, Min J, Li L et al. Reversing chemoresistance by small molecule inhibition of the translation initiation complex elF4F. Proc Natl Acad Sci USA 2011; 108: 1046-1051.

12. Herbert TP, Fahraeus R, Prescott A, Lane DP, Proud CG. Rapid induction of apoptosis mediated by peptides that bind initiation factor elF4E. Curr Biol 2000; 10: 793-796.

13. Moerke NJ, Aktas H, Chen H, Cantel S, Reibarkh MY, Fahmy A et al. Small-molecule inhibition of the interaction between the translation initiation factors elF4E and elF4G. Cell 2007; 128: 257-267.

14. Gosselin P, Martineau Y, Morales J, Czjzek M, Glippa V, Gauffeny I et al. Tracking a refined elF4E-binding motif reveals Angel1 as a new partner of elF4E. Nucleic Acids Res 2013; 41: 7783-7792.

15. Altmann M, Schmitz N, Berset C, Trachsel H. A novel inhibitor of cap-dependent translation initiation in yeast: p20 competes with elF4G for binding to elF4E. EMBO $J$ 1997; 16 : 1114-1121.

16. Mader S, Lee H, Pause A, Sonenberg N. The translation initiation-factor Eif-4e binds to a common motif shared by the translation factor Eif-4-gamma and the translational repressors 4e-binding proteins. Mol Cel Biol 1995; 15: 4990-4997.

17. Oulhen N, Boulben S, Bidinosti M, Morales J, Cormier P, Cosson B. A variant mimicking hyperphosphorylated $4 \mathrm{E}-\mathrm{BP}$ inhibits protein synthesis in a sea urchin cell-free, capdependent translation system. PLOS One 2009; 4: e5070.

18. Dong C, Li Q, Lyu SC, Krensky AM, Clayberger C. A novel apoptosis pathway activated by the carboxyl terminus of p21. Blood 2005; 105: 1187-1194.

19. Fletcher CM, McGuire AM, Gingras AC, Li H, Matsuo H, Sonenberg N et al. 4E binding proteins inhibit the translation factor elF4E without folded structure. Biochemistry 1998; 37 : 9-15.

20. Ankarcrona M, Dypbukt JM, Bonfoco E, Zhivotovsky B, Orrenius S, Lipton SA et al. Glutamate-induced neuronal death: a succession of necrosis or apoptosis depending on mitochondrial function. Neuron 1995; 15: 961-973.

21. Tsujimoto Y. Apoptosis and necrosis: intracellular ATP level as a determinant for cell death modes. Cell Death Differ 1997; 4: 429-434.

22. Barros LF, Kanaseki T, Sabirov R, Morishima S, Castro J, Bittner CX et al. Apoptotic and necrotic blebs in epithelial cells display similar neck diameters but different kinase dependency. Cell Death Differ 2003; 10: 687-697.

23. Kroemer G, Galluzzi L, Vandenabeele P, Abrams J, Alnemri ES, Baehrecke EH et al. Classification of cell death: recommendations of the Nomenclature Committee on Cell Death 2009. Cell Death Differ 2009; 16: 3-11.

24. Degterev A, Huang Z, Boyce M, Li Y, Jagtap P, Mizushima N et al. Chemical inhibitor of nonapoptotic cell death with therapeutic potential for ischemic brain injury. Nat Chem Biol 2005; 1: 112-119.

25. Cunningham CC. Actin polymerization and intracellular solvent flow in cell surface blebbing. J Cell Biol 1995; 129: 1589-1599.

26. Franklin-Tong VE, Gourlay CW. A role for actin in regulating apoptosis/programmed cell death: evidence spanning yeast, plants and animals. Biochem J 2008; 413: 389-404.

27. Smart FM, Edelman GM, Vanderklish PW. BDNF induces translocation of initiation factor $4 E$ to mRNA granules: evidence for a role of synaptic microfilaments and integrins. Proc Natl Acad Sci USA 2003; 100: 14403-14408.

28. Willett M, Brocard M, Davide A, Morley SJ. Translation initiation factors and active sites of protein synthesis co-localize at the leading edge of migrating fibroblasts. Biochem $\mathrm{J} 2011$; 438: 217-227.

29. Shankar J, Messenberg A, Chan J, Underhill TM, Foster LJ, Nabi IR. Pseudopodial actin dynamics control epithelial-mesenchymal transition in metastatic cancer cells. Cancer Res 2010; 70: 3780-3790

30. Liu L, Chen L, Chung J, Huang S. Rapamycin inhibits F-actin reorganization and phosphorylation of focal adhesion proteins. Oncogene 2008; 27: 4998-5010. 
31. Fan S, Li Y, Yue P, Khuri FR, Sun SY. The elF4E/elF4G interaction inhibitor 4EGI-1 augments TRAIL-mediated apoptosis through C-FLIP Down-regulation and DR5 induction independent of inhibition of cap-dependent protein translation. Neoplasia 2010; 12: 346-356.

32. Le Bouffant R, Boulben S, Cormier P, Mulner-Lorillon O, Belle R, Morales J. Inhibition of translation and modification of translation factors during apoptosis induced by the DNAdamaging agent MMS in sea urchin embryos. Exp Cell Res 2008; 314: 961-968.

33. Legagneux V, Omilli F, Osborne HB. Substrate-specific regulation of RNA deadenylation in Xenopus embryo and activated egg extracts. RNA 1995; 1: 1001-1008.

34. Milhas D, Cuvillier O, Therville N, Clave P, Thomsen M, Levade T et al. Caspase-10 triggers Bid cleavage and caspase cascade activation in FasL-induced apoptosis. J Biol Chem 2005; 280: 19836-19842.
Cell Death and Disease is an open-access journal published by Nature Publishing Group. This work is licensed under a Creative Commons Attribution 4.0 International Licence. The images or other third party material in this article are included in the article's Creative Commons licence, unless indicated otherwise in the credit line; if the material is not included under the Creative Commons licence, users will need to obtain permission from the licence holder to reproduce the material. To view a copy of this licence, visit http://creativecommons.org/licenses/by/4.0

Supplementary Information accompanies this paper on Cell Death and Disease website (http://www.nature.com/cddis) 